\title{
Can mild anaemia increase the risk of complications in patients over 65 years of age compared to younger patients undergoing cardiac surgery?
}

\author{
Anetta M. Kowalczuk-Wieteska, Iwona Majchrzyk, Marian Zembala, Michał Zembala \\ Chair and Clinical Department of Cardiac Surgery, Transplantology, Vascular and Endovascular, Medical University of Silesia, Zabrze, Poland
}

Adv Interv Cardiol 2020; 16, 1 (59): 116-119

DOI: https://doi.org/10.5114/aic.2020.93921

According to the World Health Organization (WHO), anaemia is a common symptom occurring in $23.9 \%$ of seniors [1]. Many retrospective studies suggest that non-cardiac surgery patients with preoperative anaemia have higher in-hospital mortality [2]. The importance of the pre-operative value of haematocrit is demonstrated by its use in the assessment of pre-operative risk on the the Society of Thoracic Surgeons score (STS) [3]. In a recent study, Kowalczuk-Wieteska et al. reported that mild anaemia (Ht 29.0-36.0\% and $\mathrm{Hb}$ 5.6-6.8 mmol/l (10$12 \mathrm{~g} / \mathrm{dl}$ ) in women and $\mathrm{Ht} 29.0-39.0 \%$ and $\mathrm{Hb}$ 5.6-7.4 $(10-13 \mathrm{~g} / \mathrm{dl})$ in men) did not increase the risk of complications in patients over 65 years of age compared to younger patients undergoing cardiac surgery.

The primary outcome was the in-hospital mortality. The secondary outcomes were as follows: paroxysmal atrial fibrillation de novo, bleeding within the chest, gastrointestinal bleeding, acute intestine necrosis, delirium, difficult wound healing of the sternum, pneumothorax, pleural liquid, haemofiltration, and intra-aortic balloon support (Table I).

The inclusion criteria involve: age $\geq 18$ years old and mild anaemia diagnosed "de novo" on admission to the cardiac surgery clinic, or at the referral centre. The exclusion criteria involve: no anaemia, anaemia more severe than mild, blood transfusions within three months before cardiac surgery, taking iron, folic acid, or vitamin $B_{12}$ preparations during a period of 3 months before surgery.

In the presented manuscript, both younger and older patients met the criteria for iron deficiency anaemia with increased inflammatory parameters, which can be classified as hospital-acquired anaemia (HAA) because $1 / 3$ of patients had an acute coronary syndrome and, related to this fact, invasive procedures (coronarography, angioplasty) using thrombolytics due to paroxysmal or fixed atrial fibrillation. In both subgroups there were no significant differences in the preoperative loads, but paroxysmal atrial fibrillation was significantly more frequent in men $(p=0.036)$, which was associated with treatment with vitamin $\mathrm{K}$ antagonist (VKA), non-vitamin $\mathrm{K}$ antagonist oral anticoagulants (NOAC), or heparin. About 50\% of patients before surgery chronically took aspirin. Sulimiery-Michalak et al. reported an over threefold increase in the ratio of anaemia chances when using both ASA and anticoagulants (VKA and NOAC) [4]. Additionally, in about $30 \%$ of patients, within 3 months before the operation, a myocardial infarction occurred, after which the patients received double antiplatelet therapy (aspirin and clopidogrel), which in $80 \%$ of elderly patients leads to gastrointestinal bleeding.

Among the limitations of the study, it should be noted that every fifth patient over 65 years old underwent a minimally invasive TAVI procedure, but no such procedure was performed in the group $<65$ years old. The two examined populations were not matched as far as the duration or complexity of the cardiosurgery procedures are concerned, because of the small number of people under 65 years old $(n=34)$, which did not allow for reliable statistical calculations.

\section{Conflict of interest}

The authors declare no conflict of interest.

\section{Corresponding author:}

Anetta M. Kowalczuk-Wieteska MD, PhD, Chair and Clinical Department of Cardiac Surgery, Transplantology, Vascular and Endovascular, Medical University of Silesia, 9 M. Curie-Skłodowskiej St, 41-800 Zabrze, Poland, phone: +48 692 645 752, e-mail: kowaletta@onet.eu Received: 10.01.2020, accepted: 22.02.2020. 
Table I. Characteristic of patients

\begin{tabular}{|c|c|c|c|c|c|c|}
\hline \multirow[t]{2}{*}{ Parameter, norm } & \multicolumn{3}{|c|}{ Before operation } & \multicolumn{3}{|c|}{ After operation } \\
\hline & $\begin{array}{l}<65 y \\
(n=34)\end{array}$ & $\begin{array}{l}\geq 65 y \\
(n=66)\end{array}$ & $P$-value & $\begin{array}{l}<65 y \\
(n=34)\end{array}$ & $\begin{array}{l}\geq 65 y \\
(n=66)\end{array}$ & $P$-value \\
\hline Age & $55.41 \pm 6.72$ & $75.32 \pm 5.81$ & $<0.001$ & $55.41 \pm 6.72$ & $75.32 \pm 5.81$ & $<0.001$ \\
\hline HT \%: & $32.73 \pm 4.01$ & $32.20 \pm 3.01$ & 0.71 & $33.51 \pm 2.57$ & $33.29 \pm 2.66$ & 0.74 \\
\hline \multicolumn{7}{|l|}{ Women: $37-47$} \\
\hline \multicolumn{7}{|l|}{ Men: 40-54 } \\
\hline $\mathrm{Hb}[\mathrm{mmol} / \mathrm{l}]:$ & $6.65 \pm 0.81$ & $6.58 \pm 0.72$ & 0.93 & $6.74 \pm 0.65$ & $6.79 \pm 0.56$ & 0.61 \\
\hline \multicolumn{7}{|l|}{ Women: 6.8-9.0 } \\
\hline \multicolumn{7}{|l|}{ Men: 7.4-9.5 } \\
\hline Erythrocytes [m/n/mm³: & $3.62 \pm 0.55$ & $3.64 \pm 0.42$ & 0.63 & $3.71 \pm 0.37$ & $3.76 \pm 0.38$ & 0.73 \\
\hline \multicolumn{7}{|l|}{ Women: $3.5-5.2$} \\
\hline \multicolumn{7}{|l|}{ Men: 4.2-5.4 } \\
\hline Iron [umol/l], 6.6-26.0 & $11.43 \pm 6.66$ & $9.93 \pm 5.28$ & 0.32 & $6.62 \pm 2.14$ & $7.99 \pm 3.85$ & 0.11 \\
\hline Ferritin [ng/ml], 15-150 & $275.56 \pm 765.31$ & $198.03 \pm 228.90$ & 0.86 & $\begin{array}{r}407.94 \\
\pm 746.03\end{array}$ & $328.88 \pm 240.5$ & 0.69 \\
\hline Transferrin [g/l], 2-3.6 & $2.65 \pm 0.69$ & $2.59 \pm 0.69$ & 0.67 & $2.14 \pm 0.44$ & $1.97 \pm 0.39$ & 0.08 \\
\hline Reticulocytes \%, 5-15 & $18.34 \pm 9.15$ & $15.44 \pm 7.35$ & 0.06 & $20.95 \pm 9.78$ & $18.11 \pm 8.20$ & 0.21 \\
\hline C-reactive protein $[\mathrm{mg} / \mathrm{l}]$ 0-5 & $15.47 \pm 29.04$ & $19.61 \pm 34.76$ & 0.56 & $67.72 \pm 70.34$ & $68.8 \pm 33.30$ & 0.89 \\
\hline $\begin{array}{l}\text { GFR glomerular filtration rate } \\
>90 \mathrm{ml} / \mathrm{min} / 1.73 \mathrm{~m}^{2}\end{array}$ & $53.41 \pm 11.16$ & $53.58 \pm 9.77$ & 0.65 & $55.02 \pm 9.32$ & $53.21 \pm 10.6$ & 0.26 \\
\hline Endocarditis (\%) & 5.88 & 4.55 & 0.85 & 5.88 & 4.55 & 0.85 \\
\hline Fa paroxysmalis (\%) & 8.82 & 19.7 & 0.25 & 17.65 & 13.64 & 0.81 \\
\hline Fa persistens & 8.82 & 19.7 & 0.25 & 8.82 & 19.7 & 0.25 \\
\hline $\begin{array}{l}\text { LICA, > } 50 \% \text { stenosis/stent/ } \\
\text { endarterectomy (\%) }\end{array}$ & 5.88 & 1.52 & 0.86 & 5.88 & 1.52 & 0.86 \\
\hline $\begin{array}{l}\text { RICA, > } 50 \% \text { stenosis/stent/ } \\
\text { endarterectomy (\%) }\end{array}$ & 2.94 & 6.06 & 0.85 & 2.94 & 6.06 & 0.85 \\
\hline Stroke (\%) & 8.82 & 7.58 & 0.86 & 0.00 & 0.00 & $<0.001$ \\
\hline TIA (\%) & 2.94 & 3.03 & 0.55 & 0.00 & 0.00 & $<0.001$ \\
\hline Delirium (\%) & 0.00 & 1.52 & 0.73 & 2.94 & 6.06 & 0.82 \\
\hline Gastrointestinal bleeding (\%) & 2.94 & 1.52 & 0.79 & 0.00 & 3.03 & 0.79 \\
\hline Intestinal diverticula (\%) & 5.88 & 1.52 & 0.79 & 5.88 & 1.52 & 0.79 \\
\hline Morbus ulcerosus (\%) & 5.88 & 10.51 & 0.68 & 5.88 & 10.51 & 0.68 \\
\hline Chronic gastritis/duodenitis (\%) & 14.71 & 4.55 & 0.17 & 14.71 & 4.55 & 0.17 \\
\hline Cirrhosis hepatis (\%) & 2.94 & 3.03 & 0.55 & 2.94 & 3.03 & 0.55 \\
\hline Rheumatoid arthritis (\%) & 2.94 & 1.52 & 0.79 & 2.94 & 1.52 & 0.79 \\
\hline Lupus erythematosus (\%) & 0.00 & 1.52 & 0.27 & 0.00 & 1.52 & 0.27 \\
\hline $\begin{array}{l}\text { Myocardial infarction < } 3 \text { months } \\
(\%)\end{array}$ & 32.35 & 28.78 & 0.89 & 32.35 & 28.78 & 0.89 \\
\hline Stent implantation < 3 months (\%) & 6.56 & 10.20 & 0.77 & 6.56 & 10.20 & 0.77 \\
\hline Active cancer disease (\%) & 8.82 & 7.58 & 0.86 & 8.82 & 7.58 & 0.86 \\
\hline
\end{tabular}


Table I. Cont.

\begin{tabular}{|c|c|c|c|c|c|c|}
\hline \multirow[t]{2}{*}{ Parameter, norm } & \multicolumn{3}{|c|}{ Before operation } & \multicolumn{3}{|c|}{ After operation } \\
\hline & $\begin{array}{l}<65 y \\
(n=34)\end{array}$ & $\begin{array}{l}\geq 65 y \\
(n=66)\end{array}$ & $P$-value & $\begin{array}{l}<65 y \\
(n=34)\end{array}$ & $\begin{array}{l}\geq 65 y \\
(n=66)\end{array}$ & $P$-value \\
\hline Aspirin (\%) & 47.06 & 51.52 & 0.83 & 47.06 & 51.52 & 0.83 \\
\hline Clopidogrel (\%) & 23.53 & 1.08 & 0.10 & 23.53 & 1.08 & 0.10 \\
\hline Brillique (\%) & 0.00 & 1.52 & 0.73 & 0.00 & 1.52 & 0.73 \\
\hline Heparin (\%) & 11.76 & 3.02 & 0.19 & 11.76 & 3.02 & 0.19 \\
\hline NOAC/VKA (\%) & 3.82 & 16.7 & 0.32 & 3.82 & 16.7 & 0.32 \\
\hline TAVI (\%) & - & - & - & 0.00 & 19.70 & 0.01 \\
\hline CABG (\%) & - & - & - & 35.29 & 16.67 & 0.86 \\
\hline OPCAB (\%) & - & - & - & 14.71 & 18.18 & 0.81 \\
\hline AVR (\%) & - & - & - & 14.71 & 16.15 & 0.73 \\
\hline AVR mini (\%) & - & - & - & 3.24 & 0.00 & 0.73 \\
\hline AVR + CABG (\%) & - & - & - & 2.94 & 10.61 & 0.34 \\
\hline RE AVR (\%) & - & - & - & 0.00 & 1.52 & 0.73 \\
\hline AVR + MV plasty (\%) & - & - & - & 0.00 & 4.55 & 0.52 \\
\hline AVR + MVR (\%) & - & - & - & 0.00 & 1.52 & 0.73 \\
\hline AVR + TV plasty (\%) & - & - & - & 0.00 & 3.03 & 0.79 \\
\hline TV plasty (\%) & - & - & - & 2.94 & 0.00 & 0.73 \\
\hline AVR + MV plasty + TV plasty (\%) & - & - & - & 2.94 & 0.00 & 0.73 \\
\hline RE TV plasty (\%) & - & - & - & 0.00 & 1.52 & 0.73 \\
\hline AAA + ASD (\%) & - & - & - & 2.94 & 0.00 & 0.73 \\
\hline TVR (\%) & - & - & - & 2.94 & 0.00 & 0.73 \\
\hline MVR + TV plasty (\%) & - & - & - & 2.94 & 0.00 & 0.73 \\
\hline Tamponade (\%) & - & - & - & 2.94 & 0.00 & 0.73 \\
\hline Sternum refixation (\%) & - & - & - & 2.94 & 0.00 & 0.73 \\
\hline ASDII (\%) & - & - & - & 2.94 & 0.00 & 0.73 \\
\hline Chest bleeding (\%) & - & - & - & 5.88 & 3.03 & 0.88 \\
\hline Acute intestine necrosis (\%) & - & - & - & 0.00 & 1.52 & 0.74 \\
\hline Complicated wounds (\%) & - & - & - & 0.00 & 1.52 & 0.74 \\
\hline Pneumothorax (\%) & - & - & - & 0.00 & 3.03 & 0.79 \\
\hline Pleural liquid (\%) & - & - & - & 8.82 & 3.03 & 0.42 \\
\hline Haemodiafiltration (\%) & - & - & - & 2.94 & 4.55 & 0.88 \\
\hline $\begin{array}{l}\text { Intra-aortic balloon pump during } \\
\text { operation (\%) }\end{array}$ & - & - & - & 0.00 & 4.55 & 0.52 \\
\hline Death $(\%)$ & - & - & - & 2.94 & 4.55 & 0.88 \\
\hline
\end{tabular}

$\mathrm{Ht}$ - haematocrit, Hb-haemoglobin, FA - atrial fibrillation, TIA - transient ischaemic attack, LICA - leftarteria carotis interna, RICA - right arteria carotis interna, $N O A C$ - novel oral anticoagulants, VKA - vitamin K antagonists, TAVI - transcatheter aortic valve implantation, OPCAB - off-pump coronary artery bypass grafting, $C A B G$ - on-pump coronary artery bypass grafting. AVR - aortic valve replacement, $R E A V R$ - reoperation aortic valve replacement, MVR - mitral valve replacement, MV plasty - mitral valve plasty, TVR - tricuspid valve replacement, TV plasty - tricuspid valve plasty, RE TV plasty - reoperation tricuspid valve plasty, ASD II - ostium secundum atrial septal defect, $A A A$ - aneurysm operation. 


\section{References}

1. Central Statistical Office of Poland/Topics/Population/Population forecast/Forecast population for 2014-2050 (compiled 2014). http://stat.gov.pl/obszarytematyczne/ludnosc/prognoza-ludnosci/prognoza-ludnosci-na-lata-2014-2050-opracowana2014r-, 1,5.html.

2. Musallam KM, Tamim HM, Richards T, et al. Preoperative anaemia and postoperative outcomes in non-cardiac surgery: a retrospective cohort study. Lancet 2011; 378: 1396-407.

3. Online STS Risk Calculator. http://riskcalc.sts.org/.

4. Sulimiera-Michalak S, Rupa-Matysek J, Gil L, et al. Comorbidities, repeated hospitalizations, and age $\geq 80$ years as indicators of anemia development in the older population. Ann Hematol 2018; 97: 1337-47. 